\title{
On a problem of Nathanson
}

\author{
Yong-Gao Chen ${ }^{1}$ and Min Tang ${ }^{2}$ \\ 1. School of Mathematical Sciences and Institute of Mathematics, \\ Nanjing Normal University, Nanjing 210023, P. R. CHINA \\ ygchen@njnu.edu.cn \\ 2. School of Mathematics and Computer Science, \\ Anhui Normal University, Wuhu 241003, P. R. CHINA \\ tmzzz2000@163.com
}

\begin{abstract}
A set $A$ of nonnegative integers is an asymptotic basis of order $h$ if every sufficiently large integer can be represented as the sum of $h$ integers (not necessarily distinct) of $A$. An asymptotic basis $A$ of order $h$ is minimal if no proper subset of $A$ is an asymptotic basis of order $h$. In this paper, we resolve a problem of Nathanson on minimal asymptotic bases of order $h$.
\end{abstract}

Keywords: minimal asymptotic basis; partition; Nathanson's problem; binary expansion

2010 Mathematics Subject Classification: 11B13

\section{Introduction}

Let $\mathbb{N}$ denote the set of all nonnegative integers and $h$ be an integer with $h \geq 2$. For $A \subseteq \mathbb{N}$ and $n \in \mathbb{N}$, let

$$
r_{h}(A, n)=\sharp\left\{\left(a_{1}, a_{2}, \ldots, a_{h}\right) \in A^{h}: a_{1}+a_{2}+\cdots+a_{h}=n\right\} .
$$

A set $A$ is called an asymptotic basis of order $h$ if $r_{h}(A, n) \geq 1$ for all sufficiently large integers $n$. In 1955, Stöhr [13] introduced the concept of minimal asymptotic basis. An asymptotic basis $A$ of order $h$ is minimal if no proper subset of $A$ is an asymptotic basis of order $h$. This means that, for any $a \in A$, the set $E_{a}=h A \backslash h(A \backslash\{a\})$ is infinite. 
In 1956, Härtter [5] proved that minimal asymptotic bases of order $h$ exist for all $h \geq 2$. Nathanson [10] constructed explicitly a minimal asymptotic basis of order 2 and an asymptotic basis of order 2 no subsets of which is minimal. Chen and Chen [1] resolved some problems on minimal asymptotic bases asked by Nathanson [11]. Jia and Nathanson [7] gave an explicit construction of minimal asymptotic bases of order $h$ for every $h \geq 2$. For related problems concerning minimal asymptotic bases, one may see [2]-[4], [6], [8]-[9] and [12].

For any nonempty subset $W$ of $\mathbb{N}$, denote by $\mathcal{F}^{*}(W)$ the set of all finite, nonempty subsets of $W$. Let $A(W)$ be the set of all numbers of the form $\sum_{f \in F} 2^{f}$, where $F \in \mathcal{F}^{*}(W)$.

In 1988, Nathanson [11] posed the following problem (see also Jia and Nathanson [7]). Problem 1. If $\mathbb{N}=W_{0} \cup \cdots \cup W_{h-1}$ is a partition such that $w \in W_{i}$ implies either $w-1 \in W_{i}$ or $w+1 \in W_{i}$, then is

$$
A=A\left(W_{0}\right) \cup \cdots \cup A\left(W_{h-1}\right)
$$

a minimal asymptotic basis of order $h$ ?

In 2011, Chen and Chen [1] obtained the following result.

Theorem A Let $h \geq 2$ and $t$ be the least integer with $t>\log h / \log 2$. Let $\mathbb{N}=W_{0} \cup \cdots \cup$ $W_{h-1}$ be a partition such that each set $W_{i}$ is infinite and contains $t$ consecutive integers for $i=1, \ldots, h$. Then

$$
A=A\left(W_{0}\right) \cup \cdots \cup A\left(W_{h-1}\right)
$$

is a minimal asymptotic basis of order $h$.

By Theorem A, the answer to Problem 1 is affirmative for $2 \leq h<4$. For $a<b$, let $[a, b]$ denote the set of all integers in the interval $[a, b]$. In this paper, the following result is proved. Thus the answer to Problem 1 is negative for $h \geq 4$.

Theorem 1. Let $h$ and $t$ be integers with $2 \leq t \leq \log h / \log 2$. Then there exists a partition $\mathbb{N}=W_{0} \cup \cdots \cup W_{h-1}$ such that each set $W_{i}$ is a union of infinitely many intervals of at least $t$ consecutive integers and

$$
A=A\left(W_{0}\right) \cup \cdots \cup A\left(W_{h-1}\right)
$$

is not a minimal asymptotic basis of order $h$.

Remark 1. For $2 \leq t<\log h / \log 2$, the following stronger result is proved: there exists a partition $\mathbb{N}=W_{0} \cup \cdots \cup W_{h-1}$ such that each set $W_{i}$ is a union of infinitely many intervals of at least $t$ consecutive integers and $n \in h A\left(W_{0}\right)$ for all sufficiently large integers $n$. 


\section{Proof of the theorem}

Since $t \geq 2$, it follows that $h \geq 2^{t} \geq 4$. For any subset $X$ of $\mathbb{N}$, let $2^{X}=\left\{2^{x}: x \in X\right\}$. Let $\left\{m_{i}\right\}_{i=1}^{\infty}$ be a sequence of integers with $m_{1}>2^{h+4}$ and $m_{i+1}-m_{i}>2^{h+4}(i \geq 1)$. Let

$$
W_{0}=\left[0, m_{1}\right] \cup\left(\bigcup_{i=1}^{\infty}\left[m_{i}+t+1, m_{i+1}\right]\right)
$$

and

$$
W_{j}=\bigcup_{\substack{i=1 \\ i \equiv j(\bmod h-1)}}^{\infty}\left[m_{i}+1, m_{i}+t\right], \quad j=1,2, \ldots, h-1
$$

It is clear that

$$
\mathbb{N}=W_{0} \cup W_{1} \cup \cdots \cup W_{h-1}
$$

If $w \in \mathbb{N} \backslash W_{0}$, then, by the definition of $W_{i}$, we have $w>m_{1}>2^{h+4}$ and $w-t \in W_{0}$. Write

$$
A=A\left(W_{0}\right) \cup \cdots \cup A\left(W_{h-1}\right) .
$$

For any positive integer, let the binary expansion of $n$ be

$$
n=\sum_{f \in F_{n}} 2^{f} .
$$

Divide into two cases according to $h>2^{t}$ and $h=2^{t}$.

Case 1: $h>2^{t}$.

In this case, we will prove that all integers $n$ with $n \geq h 2^{h(2 t+1)}$ are in $h A\left(W_{0}\right)$. Thus $A$ is not a minimal asymptotic basis of order $h$.

Let $n \geq h 2^{h(2 t+1)}$. Now we split terms in the summation of (1). First, we split all $2^{f}$ with $f \in F_{n} \backslash W_{0}$ into $2^{t}$ terms $2^{f-t}$. Then all terms are in $2^{W_{0}}$ and each term repeats at most $2^{t}+1$ times in the summation. We continue to split terms in the summation. For any term $2^{w}$ in the summation, if $w>2 t+1$ and none of $2^{w-i}(1 \leq i \leq 2 t+1)$ appears in the summation, we split $2^{w}$ (split one of $2^{w}$ if there are several such terms) as follows:

(a) $2^{w}=2^{w-1}+2^{w-1}$ if $w-1 \in W_{0}$;

(b) $2^{w}=\left(2^{t}+1\right) 2^{w-t-1}+\cdots+\left(2^{t}+1\right) 2^{w-2 t+1}+\left(2^{t}+1\right) 2^{w-2 t}+2 \times 2^{w-2 t-1}$ if $w-1 \notin W_{0}$.

In Case (b), by the definition of $W_{0}$ and $w \in W_{0}$, we know that the integers $w-t-i$ $(1 \leq i \leq t+1)$ are all in $W_{0}$.

Since each split increases the number of terms at least one, the splitting procedure must be terminated in finite steps. In the final summation, all terms are in $2^{W_{0}}$ and each term 
repeats at most $2^{t}+1$ times. If $2^{w}$ appears, then at least one of $2^{w-i}(1 \leq i \leq 2 t+1)$ appears. Let the final summation be

$$
n=\sum_{j=1}^{s} 2^{w_{s}}
$$

with $0 \leq w_{1} \leq w_{2} \leq \cdots \leq w_{s}$. Let $w_{0}=0$. Thus

$$
0 \leq w_{i+1}-w_{i} \leq w_{i+1}-\left(w_{i+1}-2 t-1\right)=2 t+1, \quad i=0,1, \ldots, s-1 .
$$

Since

$$
h 2^{h(2 t+1)} \leq n=\sum_{j=1}^{s} 2^{w_{s}} \leq\left(2^{t}+1\right) \sum_{w=0}^{w_{s}} 2^{w}=\left(2^{t}+1\right)\left(2^{w_{s}+1}-1\right)<h 2^{w_{s}+1}
$$

it follows that $w_{s} \geq h(2 t+1)$. On the other hand,

$$
w_{s}=\sum_{i=0}^{s-1}\left(w_{i+1}-w_{i}\right) \leq s(2 t+1) .
$$

Hence $s \geq h$. Noting that $2^{t}+1 \leq h$ and $s \geq h$, we can split the final summation into $h$ nonempty sums such that all terms in each nonempty sum are distinct. So $n \in h A\left(W_{0}\right)$.

Case 2: $h=2^{t}$.

It is clear that $4 \in A\left(W_{0}\right)$. Now we prove that $E_{4}=h A \backslash h(A \backslash\{4\})$ is a finite set. Thus $A$ is not a minimal asymptotic basis of order $h$.

Let $n>m_{2}$. We will show that $n \in h(A \backslash\{4\})$. That is, $n \notin E_{4}$. Divide into the following subcases:

Subcase 2.1: $F_{n} \cap W_{0} \neq\{2\}$.

Subcase 2.1.1: $F_{n} \cap W_{0} \neq \emptyset$ and $\left|F_{n} \backslash W_{0}\right| \geq h-1$. Then $F_{n} \backslash W_{0}$ has a partition

$$
F_{n} \backslash W_{0}=L_{1} \cup L_{2} \cup \cdots \cup L_{h-1}
$$

where $L_{i} \neq \emptyset(1 \leq i \leq h-1)$ and for every $L_{i}$ there exists a $W_{j}(j \geq 1)$ with $L_{i} \subseteq W_{j}$. Let $L_{0}=F_{n} \cap W_{0}$ and

$$
a_{i}=\sum_{l \in L_{i}} 2^{l}, \quad 0 \leq i \leq h-1
$$

Then

$$
a_{i} \in A \backslash\{4\}, \quad 0 \leq i \leq h-1
$$

and

$$
n=a_{0}+\cdots+a_{h-1} .
$$


Hence $n \in h(A \backslash\{4\})$.

Subcase 2.1.2: $F_{n} \cap W_{0} \neq \emptyset$ and $1 \leq\left|F_{n} \backslash W_{0}\right| \leq h-2$. Let

$$
F_{n} \backslash W_{0}=\left\{f_{0}, \ldots, f_{l-1}\right\}
$$

with $f_{0}>\cdots>f_{l-1}$. Then $f_{0} \geq m_{1}+1>2^{h+4}$. Let

$$
f_{i}=f_{0}-(i-l+1), \quad l \leq i \leq h-2
$$

and $f_{h-1}=f_{h-2}$. Put

$$
a_{0}=\sum_{f \in F_{n} \cap W_{0}} 2^{f}, \quad a_{i}=2^{f_{i}}, \quad 1 \leq i \leq h-1 .
$$

Since

$$
f_{l}>f_{l+1}>\cdots>f_{h-2}=f_{h-1}>2^{h+4}-(h-2-l+1) \geq 2^{h+4}-(h-2)>2,
$$

it follows that

$$
a_{i} \in A \backslash\{4\}, \quad 0 \leq i \leq h-1
$$

and

$$
n=a_{0}+\cdots+a_{h-1} .
$$

Hence $n \in h(A \backslash\{4\})$.

Subcase 2.1.3: $F_{n} \cap W_{0} \neq \emptyset$ and $F_{n} \backslash W_{0}=\emptyset$. That is, $F_{n} \subseteq W_{0}$. Let

$$
F_{n}=\left\{g_{0}, \ldots, g_{k-1}\right\}
$$

with $g_{0}>\cdots>g_{k-1}$. Since

$$
n>m_{2}>2^{h+4}>1+2+2^{2}+\cdots+2^{h+3},
$$

we have $g_{0} \geq h+4$.

If $k=1$, then $F_{n}=\left\{g_{0}\right\}$. Let

$$
a_{i}=2^{g_{0}-i-1}, \quad 0 \leq i \leq h-2
$$

and $a_{h-1}=a_{h-2}$. Since

$$
a_{0}>a_{1}>\cdots>a_{h-2}=a_{h-1}=2^{g_{0}-h+1}>4,
$$


it follows that

$$
a_{i} \in A \backslash\{4\}, \quad 0 \leq i \leq h-1
$$

and

$$
n=a_{0}+\cdots+a_{h-1} .
$$

Hence $n \in h(A \backslash\{4\})$.

If $k \geq 2$ and $g_{1}>2$, then we take

$$
\begin{gathered}
a_{0}=2^{g_{1}}+\cdots+2^{g_{k-1}}, \\
a_{i}=2^{g_{0}-i}, \quad 1 \leq i \leq h-2
\end{gathered}
$$

and $a_{h-1}=a_{h-2}$. Since

$$
a_{1}>\cdots>a_{h-2}=a_{h-1}=2^{g_{0}-h+2}>4,
$$

it follows that

$$
a_{i} \in A \backslash\{4\}, \quad 0 \leq i \leq h-1
$$

and

$$
n=a_{0}+\cdots+a_{h-1} .
$$

Hence $n \in h(A \backslash\{4\})$.

If $k \geq 2$ and $g_{1}=2$, then we take

$$
a_{0}=2^{g_{0}-h+1}+2^{g_{1}}+\cdots+2^{g_{k-1}}
$$

and

$$
a_{i}=2^{g_{0}-i}, \quad 1 \leq i \leq h-1 .
$$

Since

$$
a_{1}>\cdots>a_{h-1}=2^{g_{0}-h+1}>4,
$$

it follows that

$$
a_{i} \in A \backslash\{4\}, \quad 0 \leq i \leq h-1
$$

and

$$
n=a_{0}+\cdots+a_{h-1} .
$$

Hence $n \in h(A \backslash\{4\})$. 
Subcase 2.2: $F_{n} \cap W_{0}=\{2\}$. By $n>m_{2}$, we have $F_{n} \backslash W_{0} \neq \emptyset$. If $f \in F \backslash W_{0}$, then $f>m_{1}>2^{h+4}$ and $f-t \in W_{0}$ (see the arguments before Case 1). Let

$$
a_{0}=2^{2}+\sum_{f \in F_{n} \backslash\{2\}} 2^{f-t}
$$

and

$$
a_{1}=\cdots=a_{h-1}=\sum_{f \in F_{n} \backslash\{2\}} 2^{f-t} .
$$

Then

$$
a_{i} \in A\left(W_{0}\right) \backslash\{4\}, \quad 0 \leq i \leq h-1
$$

and

$$
n=a_{0}+\cdots+a_{h-1}
$$

by $h=2^{t}$. Hence

$$
n \in h\left(A\left(W_{0}\right) \backslash\{4\}\right) .
$$

This completes the proof of Theorem 1.

Acknowledgments. The authors are supported by the National Natural Science Foundation of China, Nos.11771211 and 11471017. The first author is also supported by the Priority Academic Program Development of Jiangsu Higher Education Institutions.

\section{References}

[1] F.J. Chen, Y.G. Chen, On minimal asymptotic bases, European J. Combin. 32 (2011) $1329-1335$.

[2] P. Erdős, M.B. Nathanson, Sets of natural numbers with no minimal asymptotic bases, Proc. Amer. Math. Soc. 70 (1978) 100-102.

[3] P. Erdős, M.B. Nathanson, Minimal asymptotic bases for the natural numbers, J. Number Theory 12 (1980) 154-159.

[4] P. Erdős, M.B. Nathanson, Minimal asymptotic bases with prescribed densities, Illinois J. Math. 32 (1988) 562-574.

[5] E. Härtter, Ein beitrag zur theorie der minimalbasen, J. Renie Angew. Math. 196 (1956) 170-204. 
[6] M. Jańczak, T. Schoen, Dense minimal asymptotic bases of order two, J. Number Theory 130 (2010) 580-585.

[7] X.D. Jia, M.B. Nathanson, A simple construction of minimal asymptotic bases, Acta Arith. 52 (1989) 95-101.

[8] J.B. Lee, A construction of minimal asymptotic bases, Period. Math. Hungar. 26 (1993) $211-218$.

[9] D.R. Ling, M. Tang, On minimal asymptotic g-adic bases, Bull. Aust. Math. Soc. 92 (2015) 374-379.

[10] M.B. Nathanson, Minimal bases and maximal nonbases in additive number theory, J. Number Theory 6 (1974) 324-333.

[11] M.B. Nathanson, Minimal bases and powers of 2, Acta Arith. 49 (1988) 525-532.

[12] M.B. Nathanson, A. Sárközy, On the maximum density of minimal asymptotic bases, Proc. Amer. Math. Soc. 105 (1989) 31-33.

[13] A. Stöhr, Gelöste und ungelöste fragen über basen der natürlichen zahlenreihe II, J. Renie Angew. Math. 194 (1955) 111-140. 